HD-THEP-93-23 rev

\title{
Sphaleron effects near the critical temperature
}

\author{
Meik Hellmund \\ Institut für Theoretische Physik der Universität Leipzig \\ Jochen Kripfganz* and Michael G. Schmidt \\ Institut für Theoretische Physik der Universität Heidelberg
}

\begin{abstract}
We discuss one-loop radiative corrections to the sphaleron-induced baryon number-violating transition rate near the electroweak phase transition in the standard model. We emphasize that in the case of a first-order transition a rearrangement of the loop expansion is required close to the transition temperature. The corresponding expansion parameter, the effective 3-dimensional gauge coupling approaches a finite $\lambda$ dependent value at the critical temperature. The $\lambda$ (Higgs mass) dependence of the 1-loop radiative corrections is discussed in the framework of the heat kernel method. Radiative corrections are small compared to the leading sphaleron contribution as long as the Higgs mass is small compared to the $\mathrm{W}$ mass. To 1-loop accuracy, there is no Higgs mass range compatible with experimental limits where washing-out of a $\mathrm{B}+\mathrm{L}$ asymmetry could be avoided for the minimal standard model with one Higgs doublet.
\end{abstract}

*Supported by Deutsche Forschungsgemeinschaft 


\section{INTRODUCTION}

Baryon number is violated by nonperturbative effects in the electroweak interaction [1]. While it is under controversial debate whether this could show up [2,3] in high energy laboratory experiments, there is a common agreement that this should give important effects at the high temperatures of the early universe [4]. Indeed the discussion of sphaleron [5] induced baryon number violation in a heat bath triggered the work on sizeable baryon number violation by the standard interactions. Actually in the hot electroweak phase without Higgs vacuum expectation value there is no sphaleron configuration, but still washing out of baryon number is expected if $B-L=0$ (which is rather natural in inflationary models without strong reheating). Thus we have the difficult problem of explaining baryon number generation [6] in a first-order electroweak phase transition [4, []]. Even if such a mechanism can be convincingly described, one still has to make sure that the baryon asymmetry generated is not washed out by sphaleron effects directly after the phase transition to the Higgs phase. At these temperatures $T$ the effective potential of the theory should already produce a sizeable Higgs vacuum expectation value $v(T)$ since a strong first order transition is required for baryon number production. This brings us to the subject of this paper, a discussion of the wash-out by sphalerons with a transition rate [4,8]

$$
\Gamma_{\not \beta} \sim \exp \left(-\frac{v(T)}{g T} S_{3}^{\text {Sphaleron }}\right)
$$

where $g$ is the gauge coupling and $S_{3}$ the 3 -dimensional sphaleron action. In the usual perturbative treatment of the effective potential for the Higgs field $\phi$ 9 14 $v(T) \neq 0$ is directly related to a radiatively generated $\left(\phi^{+} \phi\right)^{3 / 2} T$-term. It also depends on the Higgs coupling $\lambda$ and hence is sensitive to the Higgs mass. Requiring the baryon asymmetry not being washed out by the sphaleron effect (eq. (1.1)), an upper bound to the Higgs mass can be derived [15]. The rough bound $m_{H}<45 \mathrm{GeV}$ is already significantly violated by experiments.

Radiative corrections [8, 16, 17], the factor in front of eq. (1.1), have to be discussed. If perturbation theory in the effective 3-dimensional gauge coupling is reliable the conclusion remains unaltered that the standard electroweak theory leads to a washing out of a previously generated baryon asymmetry (if $B-L=0$ ) after the phase transition. Because of the magnitude of the effective coupling, this perturbative expansion is problematic for $m_{H}$ near $M_{W}$ close to the phase transition, whereas for smaller values of $m_{H}$ it is more trustworthy. In any case, nonperturbative effects could be responsible for a stronger first order transition, as suggested by numerical lattice studies [18,19]. If the problem of washing out the baryon asymmetry could be avoided, CP violation might be strong enough to explain the baryon number of the universe even in the standard model with a single doublet [20]. Otherwise, variants of the standard electroweak theory would have to be considered [7,21]. Also in these models, however, a thorough discussion of the baryon number violating rate including radiative effects is necessary if one wants to give quantitative bounds for the Higgs mass.

One might doubt whether the perturbative treatment of the effective action in the temperature range close to the phase transition and with field configurations restricted by the sphaleron ansatz is reliable. There are several dangerous aspects we should shortly mention at the beginning and comment on in more detail later on. 
The region around the Higgs field $\phi=0$ has strong infrared effects like those in QCD and one has to inspect how sensitive to this region the sphaleron calculation is. As argued in ref. [13], infrared divergences due to Goldstone bosons also appear for $\phi \sim v(T)$ in the selfconsistency (gap type) equations if the Landau gauge is used. We do not expect these singularities to be physical.

The sphaleron is a 3 -dimensional object, and it is consequent to discuss it with an effective action where the nonzero Matsubara frequencies are already integrated out, i. e. in a 3-dimensional theory for the static modes. In low order perturbation theory, combined with a high temperature expansion this can be carried out. The non-static modes are expected to behave perturbatively, except for plasma mass effects, whereas the static (zero) modes may show non-perturbative behaviour (dynamical mass generation, condensate formation, confinement). It is convenient to use the high temperature expansion in integrating the non-static modes. However, the phase transition may be at temperatures where this is not valid anymore.

It is only partly a matter of convenience which radiative corrections should already be included in the effective action which gives the sphaleron configuration. In principle one could use "exact" renormalization group techniques [22.223] to arrive at the appropriate action at some scale, but even the discussion of one-loop corrections to the fundamental Lagrangian with the well-known sphaleron gives interesting insights.

The 3-dimensional effective (dimensionless) gauge coupling $g_{3}^{2}(T)=\frac{g \cdot T}{v(T)}$ is not very small at the temperatures of the supposed first order phase transition (but does not diverge as for second-order transitions). This supports the high temperature expansion, but causes problems with the perturbative expansion. Though it would be certainly more satisfying to see all these aspects controlled in a genuinely nonperturbative treatment, a perturbative approach might still give a good orientation.

When this paper was first written [24], an exact calculation of the 1-loop sphaleron determinant by Carson et al. [17] had been available for relatively large values of the Higgs mass. The result apparently differed considerably from the answer one would get in determining the sphaleron using the 1-loop effective potential. Later, Baacke and Junker 25] presented another complete calculation of the determinant, with rather different results. In the mean-time, some errors have been corrected, and the use of different schemes has been clarified by these authors [26]. Both 'exact' answers are now essentially consistent, and are furthermore surprisingly well represented up to intermediate Higgs masses by the $\phi^{3}$ term of the 1-loop effective potential. The results of refs. [17,26] cannot directly be used near the electroweak phase transition because the original expansion parameter becomes infinite. The loop expansion has to be rearranged. The $\lambda$ dependence of the 1-loop corrections will be estimated using the heat-kernel expansion. These will be the main subjects of this paper.

Chapter 2 contains a discussion of the effective three-dimensional action including the $\phi^{3}$-term at temperatures close to the phase transition. In chapter 3 we study the radiative corrections to the baryon number violating transition rate induced by the sphaleron. The $\lambda$ (Higgs mass) dependence of the different parts of the rate are discussed in detail. Using the 't Hooft-Feynman gauge plays an important role. Chapter 4 contains numerical evaluations and a discussion of our results. 


\section{THE ONE-LOOP EFFECTIVE ACTION NEAR THE PHASE TRANSITION}

The effective action for the sphaleron saddle point [5] can differ from the fundamental action. The inclusion of 1-loop effects (eventually enlarged by the selfconsistent IR plasma mass corrections) has important effects in temperature quantum field theory, in particular the $T^{2} \phi^{2}$ plasma mass term. There is also a $T$-dependent coupling $\lambda_{T}$ and there will appear new terms in the action like $\phi^{6} / T^{2}$ etc. if one completely integrates our the nonzero Matsubara frequencies. The latter terms are neglected in the following in the spirit of a high temperature expansion.

After a rescaling

$$
r \rightarrow \frac{\xi}{g v_{0}(T)}, \quad A_{\mu} \rightarrow v_{0}(T) A_{\mu}, \quad \phi \rightarrow v_{0}(T) \phi
$$

the electroweak standard action (without Weinberg mixing) including plasma mass and $\lambda_{T}$, and reduced to three dimensions can be written as [16, 17]

$$
\begin{aligned}
\frac{1}{g_{3}^{(0) 2}(T)} S_{3}^{(0)}= & \frac{1}{g_{3}^{(0) 2}(T)} \int d^{3} \xi\left[\frac{1}{4} F_{i k}^{a} F_{i k}^{a}+\left(D_{i} \phi\right)^{+}\left(D_{i} \phi\right)\right. \\
& +\frac{\lambda_{T}}{g^{2}}\left(\phi^{+} \phi-\frac{1}{2}\right)^{2} \\
& \left.+\frac{1}{2} A_{0}\left(-D_{i} D_{i}+\frac{1}{2} \phi^{+} \phi\right) A_{0}\right]
\end{aligned}
$$

with the effective 3-dimensional gauge coupling

$$
g_{3}^{(0) 2}(T)=\frac{g T}{v_{0}(T)}
$$

and with T-dependent minimum of the potential (in the notation of ref. [9])

$$
v_{0}^{2}(T)=\frac{2}{\lambda_{T}}\left(T_{0}^{2}-T^{2}\right) D
$$

where

$$
\begin{aligned}
D & =\frac{1}{8 v_{0}^{2}}\left(3 m_{W}^{2}+2 m_{t}^{2}\right) \quad\left(v_{0}=246 G e V\right) \\
T_{0}^{2} & =\frac{m_{H}^{2}-8 v_{0}^{2} B}{4 D} \\
m_{W}^{2} & =\frac{1}{4} g^{2} v_{0}^{2}, \quad m_{H}^{2}=2 \lambda v_{0}^{2} \\
\lambda_{T} & =\lambda-\frac{3}{16 \pi^{2} v_{0}^{4}}\left(3 M_{W}^{4} \log \frac{M_{W}^{2}}{a_{B} T^{2}}-4 m_{t}^{4} \log \frac{m_{t}^{2}}{a_{F} T^{2}}\right) \\
B & =\frac{3}{64 \pi^{2} v_{0}^{4}}\left(3 m_{W}^{4}-4 m_{t}^{4}\right) \\
\log a_{B} & =2 \log 4 \pi-2 \gamma \\
\log a_{F} & =2 \log \pi-2 \gamma
\end{aligned}
$$


This would predict a second-order phase transition at $T \rightarrow T_{0}$, where $v_{0}(T) \rightarrow 0$ and hence $g_{3}^{(0) 2}(T) \rightarrow \infty$. The saddle point approximation would break down close to $T_{0}$, as well as the perturbative expansion in $g_{3}^{(0) 2}$. Discussing the baryon asymmetry a first order phase transition is required and indeed perturbation theory provides us with a term $T\left(g^{2} \phi^{+} \phi\right)^{\frac{3}{2}}$ in the effective potential suggesting a first-order phase transition. This might be as misleading as in the pure $\phi^{4}$ theory where the coupling $\lambda$ appearing in this term vanishes [27 at the phase transition. However, the latter does not happen with the 4-dimensional gauge coupling $g$. The term above is a zero-mode 3-dimensional IR effect

$$
V\left(m^{2}\right) \sim\left(m^{2}\right)^{\frac{3}{2}} T
$$

with $m^{2}=\frac{g^{2} \phi^{+} \phi}{2}$ for gauge bosons.

There are of course further contributions to the effective action (2.2), among them a Debye mass term

$$
\frac{1}{2}\left(\frac{1}{6}\left(5+N_{F}\right) g^{2} T^{2}\right) A_{0}^{a} A_{0}^{a}
$$

Because of this longitudinal gauge boson plasma mass ${ }^{2} \sim T^{2}$ their contribution is suppressed at high temperatures. There is also a mass counter term depending linearly on the cut-off. It arises because the 4-dimensional one-loop integrals leave out the static modes and cancel against divergences of the 3 -dimensional theory.

Thus we include a term $-E\left(2 \phi^{+} \phi\right)^{\frac{3}{2}}$ in the potential with

$$
E=\frac{2}{3} \frac{3}{32 \pi} g^{3}
$$

where the factor $\frac{2}{3}$ is due to the suppression of the longitudinal gauge boson in eq. (2.2) [9]. Following the discussion [9,10] this leads to a first-order transition to a new minimum

$$
v(T)=\frac{3}{2} \frac{E T}{\lambda_{T}}+\sqrt{\left(\frac{3}{2} \frac{E T}{\lambda_{T}}\right)^{2}+v_{0}^{2}(T)}
$$

where $v_{0}(T)$ is given in eq. (2.4). The critical temperature where both minima of the potential are equal is at

$$
T_{c}^{2}=\frac{T_{0}^{2}}{1-\frac{E^{2}}{\lambda_{T_{c}} D}}
$$

where $v\left(T_{c}\right)=\frac{2 E T_{c}}{\lambda_{T_{c}}}$, and classical instability at $\phi=0$ sets in at $T=T_{0}$ where $v\left(T_{0}\right)=$ $\frac{3 E T_{0}}{\lambda_{T_{0}}}$.

If we rescale with $v(T)$ instead of $v_{0}(T)$, we obtain an effective action like (2.2) with

$$
g_{3}^{2}(T)=\frac{g T}{v(T)}
$$

but with a modified effective potential 


$$
\begin{aligned}
V_{\text {eff }}= & \frac{1}{g_{3}^{2}(T)} \frac{\lambda_{T}}{g^{2}} \int d^{3} \xi\left[\left(\phi^{+} \phi-\frac{1}{2}\right)^{2}+\epsilon\left(\frac{3}{4}\left(\phi^{+} \phi-\frac{1}{2}\right)\right.\right. \\
& \left.\left.-\frac{1}{\sqrt{2}}\left(\left(\phi^{+} \phi\right)^{\frac{3}{2}}-\left(\frac{1}{2}\right)^{3 / 2}\right)\right)\right] .
\end{aligned}
$$

The second part is related to the first one by a new parameter $\epsilon$, on which the quasiclassical solution will depend.

$$
\epsilon(T)=\frac{4}{3}\left(1-\frac{v_{0}^{2}(T)}{v^{2}(T)}\right)=\frac{8}{3+\left(1+8 \frac{T_{0}^{2}\left(T_{c}^{2}-T^{2}\right)}{T^{2}\left(T_{c}^{2}-T_{0}^{2}\right)}\right)^{\frac{1}{2}}}
$$

which is $\epsilon=2$ for $T=T_{c}, \epsilon=\frac{4}{3}$ for $T=T_{0}$, and (formally) $\epsilon=0$ for $T=0$. Thus in the region of the phase transition $\epsilon \sim 0(1)$ the second term has a prefactor of similar size as the first one. There are two expansion parameters $\frac{g_{3}^{2}(T)}{4 \pi}$ and $\frac{\lambda_{T}}{g^{2}}$, varying independently through $\mathrm{T}$ and $\lambda$. According to

$$
\frac{g_{3}^{2}(T)}{4 \pi}=\frac{\lambda_{T}}{g^{2}} \frac{g^{3}}{16 \pi E} \epsilon=\frac{\lambda_{T}}{g^{2}} \epsilon
$$

they are of about equal size close to the phase transition. The effective gauge coupling $g_{3}^{2}(T)$ is kept small with small $\frac{\lambda_{T}}{g^{2}}$, which is the case for small Higgs mass. However, due to a heavy top quark,$\frac{\lambda_{T}}{g^{2}}$ cannot be arbitrarily small. For $m_{\text {top }}=150 \mathrm{GeV}$ and $m_{\text {top }}=200 \mathrm{GeV}$ it is bounded from below by 0.032 and 0.08 , resp., following eq. (2.5). The relation between $\frac{\lambda_{T}}{g^{2}}$ and $\frac{m_{H}^{2}}{M_{W}^{2}}$ at $T=T_{0}$ is shown in Fig. 1.

In the case $m_{H}^{2} \sim M_{W}^{2} g_{3}^{2}\left(T_{c}\right)$ is of order one, and it is not clear a priori whether this is small enough for the quasiclassical expansion (in the broken symmetry phase) to be well behaved.

The high temperature expansion breaks down if $g_{3}^{2}(T)$ is too small:

$$
\frac{M_{W}(T)}{T}=\frac{g v(T)}{2 T}=\frac{g^{2}}{2 g_{3}^{2}(T)}=\frac{2 g^{2}}{\left(\lambda_{T} / g^{2}\right)} \frac{1}{16 \pi \epsilon}
$$

This can only be avoided in the limit $g \rightarrow 0, \frac{\lambda_{T}}{g^{2}}$ fixed. Assuming a limit of validity of the high temperature expansion of $\frac{M_{W}(T)}{T}<2$, eq. (2.13) requires $g_{3}^{2}(T)>\frac{g^{2}}{4}$. For a large top mass the requirement $\frac{m_{t}(T)}{T}<2$ is more restrictive but in this case an exact treatment of the temperature dependence is no problem.

The 3-dimensional reduction based on the dominance of zero modes, crucial for the $\phi^{3} T$ term requires $2 \pi T \gg M_{W}(T)$ or $\pi T>m_{t}(T)$, respectively, which is less restrictive than the previous inequality.

\section{ONE-LOOP FLUCTUATIONS AROUND THE SPHALERON}

The transition rate 28,29 induced by the sphaleron (responsible for a possible wash-out of a baryon asymmetry) is $8,16,30,31$ 


$$
\begin{aligned}
\Gamma / V= & \frac{\omega_{-}}{2 \pi} N_{\text {trans }}(N V)_{\text {rot }}\left(\frac{g^{2} T}{(4 \pi)^{2}}\right)^{3}\left(\frac{g_{3}^{2}}{4 \pi}\right)^{-6} \\
& \times \exp \left(-\frac{1}{g_{3}^{2}(T)} S_{3}^{(0)}-S_{3}^{(1, \alpha)}-S_{3}^{(1, \beta)}\right)
\end{aligned}
$$

Here $\omega_{-}^{2}$ is the eigenvalue of the unstable mode and as usual the integration over collective coordinates corresponding to translational and rotational zero modes is taken out explicitly. $S_{3}^{(0)}$ is the classical sphaleron action, and $S_{3}^{(1, \alpha)}$ that part of the one-loop action obtained from the $\phi^{3}$ effective potential and the corresponding shift of $g_{3}^{2}(T)$

$$
S_{3}^{(1, \alpha)}=\tilde{E} \int d^{3} \xi\left(\frac{3}{4}\left(\phi^{+} \phi-\frac{1}{2}\right)-\frac{1}{\sqrt{2}}\left(\left(\phi^{+} \phi\right)^{\frac{3}{2}}-\left(\frac{1}{2}\right)^{3 / 2}\right)\right)
$$

with $\tilde{E}=\frac{4 E}{g^{3}}=\frac{1}{4 \pi}$ according to eq. (2.12). $S_{3}^{(1, \beta)}$ contains the remaining 1-loop contribution, i. e. the full 1-loop action with the $\phi^{3}$ term subtracted

$$
S_{3}^{(1, \beta)}=-\left(\log \kappa-\frac{\tilde{E}}{\sqrt{2}} \int d^{3} \xi\left(\left(\phi^{+} \phi\right)^{\frac{3}{2}}-\left(\frac{1}{2}\right)^{3 / 2}\right)\right.
$$

$\kappa$ is given by

$$
\kappa=\operatorname{Im}\left(\frac{\operatorname{det}^{\prime}\left(\frac{\delta^{2} S_{g f}}{\delta \phi^{2}}\right)_{\phi=\phi_{v a c}}}{\operatorname{det}^{\prime}\left(\frac{\delta^{2} S_{g f}}{\delta \phi^{2}}\right)_{\phi=\phi_{s p}}}\right)^{1 / 2}\left(\frac{\Delta_{F P \phi=\phi_{s p}}}{\Delta_{F P \phi=\phi_{v a c}}}\right)
$$

We follow the notation of ref. [16,17]. The prime in eq. (3.4) indicates that zero modes have been removed from the sphaleron determinant and a corresponding number of low-lying eigenvalues are dropped in the free field determinant. $\kappa$ still contains the unstable mode contribution, which also has to be taken out of the determinant explicitly. We discuss this fluctuation determinant in the framework of the heat-kernel expansion. In the Schwinger proper time formulation one has

$$
\log \frac{\operatorname{det} K}{\operatorname{det} K_{0}}=\lim _{\epsilon \rightarrow 0}\left(-t r \int_{\epsilon}^{\infty} \frac{d t}{t}\left(e^{-t K}-e^{-t K_{0}}\right)\right.
$$

At large $t$, the integrand is well approximated by the lowest eigenstates in the spectrum. For $m_{H}^{2} \sim m_{W}^{2}$ there is only one scale in the theory, both in the vacuum sector (" $K_{0}$ ") and the sphaleron sector (" $K$ "), and the large $t$ behaviour can be well parametrized by a form $A e^{-t m_{W}^{2}}$ after subtracting the zero modes and the unstable mode which have to be taken out explicitly. In numerical evaluations [16] the subtraction of the exponentially increasing mode contribution is very problematic and deserves further studies.

Because of the exponential damping, the main part of the integral comes from small $t m_{W}^{2}$ where an expansion of the exponentials in (3.5) in $t$ makes sense, though the radius of convergence is not known. Such a t expansion has been carried out in ref. [16]. The expression (3.5) can be transformed into 


$$
\begin{aligned}
\log \frac{\operatorname{det} K}{\operatorname{det} K_{0}}= & -\int_{0}^{\infty} \frac{d t}{t} \int d^{d} x \int \frac{d^{d} p}{(2 \pi)} e^{-t p^{2}} \\
& \times \operatorname{tr}\left(e^{-t \delta K}-e^{-t \delta K_{0}}\right)
\end{aligned}
$$

with

$$
\begin{aligned}
& \delta K(p, x)=-2 i p \cdot D(A)-(D(A))^{2}+V \\
& \delta K(p, x)=-2 i p \partial-\partial^{2}+V_{0}
\end{aligned}
$$

In the present case, an explicit expansion in $t$ can be obtained. $V$ is a $13 \times 13$ matrix (or a $3 \times 3$ matrix for the ghosts). After $p$-integration, the authors of ref. [16] find $(d=3)$

$$
\log \frac{\operatorname{det} K}{\operatorname{det} K_{0}}=-\int_{0}^{\infty} \frac{d t}{t} \frac{1}{(4 \pi t)^{d / 2}} \sum_{p=1}^{\infty} \frac{(-t)^{p}}{p !} O_{p}
$$

with the first three operators $O_{p}, p=1,2,3$ given explicitly in ref. [16]. Since we use a different rescaling, our fluctuation operator differs from that of ref. [16] in replacing $\left(\phi^{+} \phi-\right.$

$\left.\frac{1}{2}\right)$ by $\left(\phi^{+} \phi-\frac{1}{2} \frac{v_{0}^{2}}{v^{2}}\right)$. This leads to corresponding modifications of prefactors of derivative operators as well.

The expansion (3.7) is appropriate for the small $t$ part of the integral. Eq. (3.7) should therefore be applied to some $t$-interval $(0, \bar{t})$ only. $\bar{t}$ should be chosen to smoothly approach the large $t$ behaviour governed by the first few eigenvalues of $K$ and $K_{0}$, respectively.

Convergent $t$ integrals are obtained if the exponential $e^{-m_{0}^{2} t}$, with $m_{0}$ the mass gap of the free theory (i. e. $K_{0}$ ) is not expanded but taken out. This leads to some operator rearrangements $O_{p} \rightarrow \tilde{O}_{p}$. This exponential fall-off does not properly describe the behaviour of $e^{-K t}$, however, which is governed by the unstable mode, various zero modes, and possible bound states at positive energy (studied in ref. [32]). After proper subtractions the first positive eigenvalue [32 of $K$ should dominate.

It is tempting to try to sum up subsets of operators to all orders. The most prominent case are operators with no derivatives. If in eq. (3.6) all derivative operators are dropped, one obtains

$$
\log \frac{\operatorname{det} K}{\operatorname{det} K_{0}}=I\left(m^{2}\right)-I\left(m_{0}^{2}\right)=-\int_{0}^{\infty} \frac{d t}{t} \int d^{3} x \int \frac{d^{3} p}{(2 \pi)^{3}} e^{-t p^{2}}\left(e^{-m^{2} t}-e^{-m_{0}^{2} t}\right)
$$

First performing the $p$ integral one finds

$$
I\left(m^{2}\right)-I\left(m_{0}^{2}\right)=-\int \frac{d t}{t} \int d^{3} x \frac{1}{(4 \pi t)^{3 / 2}} e^{-m_{0}^{2} t}\left(e^{\left(m_{0}^{2}-m^{2}\right) t}-1\right)
$$

Expanding the last factor in $\mathrm{t}$ and performing the $t$ integration leads to

$$
I\left(m^{2}\right)-I\left(m_{0}^{2}\right)=-\left(\frac{m_{0}^{2}}{4 \pi}\right)^{3 / 2} \sum_{p=1}^{\infty} \frac{\Gamma(p-3 / 2)}{\Gamma(p+1)} \int d^{3} x\left(\frac{m_{0}^{2}-m^{2}}{m_{0}^{2}}\right)^{p}
$$

This procedure automatically regularizes the UV divergence originally present (for $p=$ $1)$. 
Eq. (3.6) can of course be evaluated directly. First performing the $t$ integral and differentiating twice with respect to $m^{2}$ in order to regularize the UV diagram, we find

$$
\begin{aligned}
I^{\prime \prime}\left(m^{2}\right) & =-\int d^{3} x \int \frac{d^{3} p}{(2 \pi)^{3}} \frac{1}{\left(p^{2}+m^{2}\right)^{2}} \\
& =-\frac{1}{8 \pi} \int d^{3} x \frac{1}{\sqrt{m^{2}}}
\end{aligned}
$$

Integrating again produces the $\phi^{3}$ term

$$
I\left(m^{2}\right)-I\left(m_{0}^{2}\right)=-\frac{1}{2 \pi} \frac{1}{3} \int d^{3} x\left(\left(m^{2}(\phi)\right)^{3 / 2}-\left(m_{0}^{2}\right)^{3 / 2}\right)
$$

Expanding around $m^{2}=m_{0}^{2}$ leads to eq. (3.10). Uncertainties due to finite renormalizations do not arise here because the linear divergence is not present in the full (4-dim) theory, and therefore no corresponding counterterms are required.

The correct normalization of the $\phi^{3}$ term is obtained by counting 9 degrees of freedom for W's, three for Goldstone bosons, one for the Higgs boson, and subtracting six for the ghosts with masses

$$
\begin{aligned}
m_{W}^{2} & =\frac{1}{2} \phi^{+} \phi \\
m_{\chi}^{2} & =\frac{1}{2} \phi^{+} \phi+\frac{2 \lambda}{g^{2}}\left(\phi^{+} \phi-\frac{1}{2} \frac{v_{0}^{2}(T)}{v^{2}(T)}\right) \\
m_{H}^{2} & =\frac{2 \lambda}{g^{2}}\left(3 \phi^{+} \phi-\frac{1}{2} \frac{v_{0}^{2}(T)}{v^{2}(T)}\right) \\
m_{g h}^{2} & =\frac{1}{2} \phi^{+} \phi
\end{aligned}
$$

respectively. In the 't Hooft-Feynman gauge the Goldstone boson is massive in the vacuum of the broken symmetry phase, in contrast to Landau gauge (and $v=v_{0}$ ).

The adjoint Higgs representation of the dimensionally reduced theory (corresponding to the $A_{0}$ field) is not taken into account because of plasma masses. Therefore, we do not find the partial cancellation between ghosts and the adjoint Higgs field (i. e. $A_{0}$ ) assumed in ref. [16]. In this way we get the properly reduced coefficient of the $\phi^{3}$ term.

If we keep the full $\lambda$ dependent masses eq.(3.13) we generate the complete 1-loop effective potential and not just the $\phi^{3}$ term

$$
\begin{aligned}
V_{\text {eff }}= & \frac{1}{g_{3}^{2}} \int d^{3} \xi\left[\frac{\lambda}{g^{2}}\left(\left(\phi^{+} \phi\right)^{2}-\frac{v_{0}^{2}}{v^{2}} \phi^{+} \phi\right)\right. \\
& -g_{3}^{2}\left\{\frac{1}{4 \pi}\left(\frac{1}{2} \phi^{+} \phi\right)^{3 / 2}\right. \\
& +\frac{1}{4 \pi}\left(\frac{1}{2} \phi^{+} \phi+\frac{2 \lambda}{g^{2}}\left(\phi^{+} \phi-\frac{1}{2} \frac{v_{0}^{2}}{v^{2}}\right)\right)^{3 / 2} \\
& \left.\left.+\frac{1}{12 \pi}\left(\frac{2 \lambda}{g^{2}}\left(3 \phi^{+} \phi-\frac{1}{2} \frac{v_{0}^{2}}{v^{2}}\right)\right)^{3 / 2}\right\}\right]
\end{aligned}
$$


In our notation, the difference to the $\phi^{3}$ term is one contribution to $S^{(1, \beta)}$

$$
\begin{aligned}
S_{V}^{(1, \beta)}= & \int d^{3} \xi\left\{\frac{1}{4 \pi}\left(\frac{1}{2} \phi^{+} \phi\right)^{3 / 2}\right. \\
& -\frac{1}{4 \pi}\left(\frac{1}{2} \phi^{+} \phi+\frac{2 \lambda}{g^{2}}\left(\phi^{+} \phi-\frac{1}{2} \frac{v_{0}^{2}}{v^{2}}\right)\right)^{3 / 2} \\
& \left.-\frac{1}{12 \pi}\left(\frac{2 \lambda}{g^{2}}\left(3 \phi^{+} \phi-\frac{1}{2} \frac{v_{0}^{2}}{v^{2}}\right)\right)^{3 / 2}\right\}
\end{aligned}
$$

to be subtracted at $\phi^{+} \phi=\frac{1}{2}$.

This contribution is well behaved above $T_{0}$. Below, it becomes complex. This already tells us that one should be reluctant in summing partial sets of operators.

One can attempt to sum further particular classes of operators, with a given number of derivatives. This would generate the derivative expansion of the effective action. The effective potential has already been discussed. As one nontrivial example we present the contribution of the field strength operator $F F$ to $S^{(1, \beta)}$

$$
S_{F F}^{(1, \beta)}=-\int d^{3} \xi\left(\frac{173}{24}\right) \frac{1}{32 \pi}\left(\frac{\phi^{+} \phi}{2}\right)^{-1 / 2} F_{i j}^{a} F_{i j}^{a}
$$

obtained from summing up the $\lambda$-independent parts of $\left(\phi^{+} \phi\right)^{n} F F$, arising from the gauge, Goldstone, and ghost loop, resp. A numerical discussion will be given in chapter 4 .

It is instructive to keep the $t$ integral in analogy to eq. (3.7). In a sphaleron background, the integrand falls off only power-like with t. These partially summed operators therefore represent a $t$-dependence very different from the true large $t$ behaviour. This causes some doubts on whether these contributions separately give a good representation of the complete effective action.

Considering high derivative operators one actually finds the t integrand increasing powerlike in $t, i$. e. the t-integrals diverge, and the derivative expansion actually breaks down for the sphaleron. The reason is that $m^{2}(\phi)$ approaches zero for $\xi \rightarrow 0$ in the sphaleron background. Therefore, the corresponding corner of the integrand is not exponentially suppressed in t. This could be interpreted as being related to the presence of the negative mode which could be build up more and more by higher derivative terms.

Partial resummation of certain operators becomes meaningful, however, in studying the leading contributions at small $\lambda$. These singularities arise from the large $\xi$ behaviour of the $\xi$-integral, because of the slow fall-off of the Higgs component of the sphaleron at small $\lambda$. At small $m_{H}$, the sphaleron has a core with size $O\left(m_{W}^{-1}\right)$. Outside the gauge field vanishes whereas the Higgs field falls off only like $e^{-m_{H} r}$. More precisely, the asymptotic behavior is

$$
\sqrt{2}|\phi| \underset{r \rightarrow \infty}{\simeq} 1-\frac{\text { const. }}{r} e^{-m_{H} r}
$$

For $m_{H} \rightarrow 0, S_{3}^{(1, \alpha)}$ will be singular because of the large $r$ behaviour. However, the leading $\frac{1}{\lambda_{T}}$ singularity cancels between the two contributions. Therefore, $S_{3}^{(1, \alpha)}$ has only a square root singularity in $\frac{1}{\lambda_{T}}$, in contrast to the $\frac{1}{\lambda_{T}}$ singularity of the $\phi^{3}$ term. 
Operators containing the gauge field strength $F_{\mu \nu}$ fall off sufficiently fast, and also terms containing two or more covariant derivatives $D_{\mu} \phi$ lead to convergent integrals. The powerlike singular contributions to the effective action are therefore just given by the effective potential.

$\lambda$ singularities associated with "bound states" in the fluctuation spectrum would only be logarithmic. If $m_{+}$denotes such a bound state mass which vanishes with $\lambda$ the corresponding

contribution from the large $\mathrm{t}$ region is proportional to $\log \frac{m_{+}^{2}}{\bar{t}}$. The $\bar{t}$-dependence will cancel against contributions from the small t region, but a logarithmic term $\log \frac{m_{+}^{2}}{m_{W}^{2}}$ could remain. Stronger $\lambda$ singularities can only arise from the small t region and should show up in the heat kernel expansion.

\section{DISCUSSION}

Necessary criteria for a well-behaved quasi-classical treatment of sphaleron transitions is that 1-loop terms are small compared to the classical sphaleron action. In the original formulation this was not the case because the coupling blows up at the critical temperature. This is cured by redefining the coupling according to the one-loop vacuum expectation value $v(T)$, as outlined in the chapter before. The question now is whether one-loop terms will be small compared to $\frac{1}{g_{3}^{2}} S_{0}$ even at the critical temperature.

Singularities in $\lambda$ arise because some of the operators appearing in the expansion become singular for $\lambda$ going to zero. Those operators are just the ones without derivatives. They are either multiplied with powers of $\lambda$, and therefore are actually non-singular, ore can be summed up to produce the $\phi^{3}$ term, i. e. cancel in $S_{3}^{(1, \beta)}$. To any finite order in the heatkernel expansion, $S_{3}^{(1, \beta)}$ will be nonsingular in $\lambda$. A separation of the 1-loop correction into $S_{3}^{(1, \alpha)}$ and $S_{3}^{(1, \beta)}$ is meaningful because of the different singularity structure in $\lambda$.

In Table \&, we present numerical results for all the operators occuring in the heat kernel expansion up to third order, i. e. as far as the expansion has been worked out in ref. [16]. This numerical study confirms that only the first few non-derivative operators show a strong $\lambda$ dependence.

To this low order in the heat kernel expansion it is not possible to give a reliable estimate of $S_{3}^{(1, \beta)}$. In particular, introducing an exponential cut-off following ref. [33] does not lead to stable results. For reliable quantitative statements on the absolute normalization of $S_{3}^{(1, \beta)}$ it will be necessary to work out more terms of the heat kernel expansion. This presumably requires the development of new techniques [34.

Since we can isolate those operators in the heat kernel expansion showing a strong $\lambda$ dependence we should understand the $\lambda$ dependence of the sphaleron rate. A complete oneloop calculation at one particular $\lambda$ value (e. g. $m_{H}=M_{W}$ ) could thus be used to normalize the rate, and predict the $\lambda$ dependence through our results.

We now present a numerical study of 1-loop corrections to the sphaleron rate. For a quick estimate, we can simply replace the Higgs potential by the 1-loop effective potential in studying the sphaleron solution. The resulting sphaleron energy is shown in Fig. 2, in terms of the profile function $A\left(\lambda_{T} / g^{2}, \epsilon\right)$ defined by

$$
\frac{E_{s p}}{T}=\frac{2 \pi}{g_{3}^{2}(T)} A\left(\lambda_{T} / g^{2}, \epsilon(T)\right)
$$


with $\epsilon(T)$ given by eq. (2.11). Even at the critical temperature, i. e. $\epsilon=2, A$ changes not more than about $10 \%$. The $T$-dependence of the sphaleron transition rate is therefore almost exclusively given by the behaviour of $g_{3}^{2}(T)$. Similar plots have been given independently in ref. [35.

For a more systematic study, one should not work with such a 'deformed' sphaleron but present the one-loop correction in terms of the original sphaleron solution. Results are shown in Fig. 3, as function of $\lambda_{T} / g^{2}$. The solid line represents the leading order contribution at $T=T_{c}$. It is only defined after reordering the loop expansion by absorbing appropriate 1-loop contributions into $g_{3}^{2}$. If this would not be done, the leading term would be zero at $T=T_{0}$, and the one-loop correction would essentially be given by the $\phi^{3}$ term (dasheddotted line in Fig. (3) in the $\lambda$ range considered. It is amusing to note that the two procedures would predict a quite similar sphaleron rate. However, in the latter case the prediction is meaningless because the expansion parameter becomes infinite.

In our scheme, the $\phi^{3}$ term does not give the leading 1-loop correction but is subtracted by a $\phi^{2}$ term with the same singularity in $\lambda$. The resulting contribution $S_{3}^{(1, \alpha)}$ (shown as dashed line in Fig. (3) is very small compared to the leading term in the whole $\lambda$ range. This is not the case for the remaining 1-loop corrections. $S_{V}^{(1, \beta)}$ is shown as dotted line in Fig. 3 . It is small at small $\lambda$ but increases and becomes of the order of the leading term for $\lambda$ larger than about 0.1. $S_{F F}^{(1, \beta)}$ shows a similar behaviour, presented in Fig. 国. Again, the correction becomes of order one for $\lambda_{T}$ about 0.1. Its sign is different from that of $S_{V}^{(1, \beta)}$, however. There are two possible conclusions one could draw at this point. One possibility is that the quasiclassical expansion breaks down for $m_{H}$ near $M_{W}$. The one-loop effective coupling is of order one in this case, and one should certainly study higher loop contributions to the effective action.

The other logical possibility would be a substantial cancellation between different terms of the derivative expansion such that the correction to the leading quasiclassical term remains small, but only the derivative expansion breaks down. Since we could not carry out the heat-kernel expansion to sufficiently high order we cannot decide this point.

For the problem of washing-out the baryon asymmetry this may not be so relevant, because the sphaleron rate is far too high in the experimentally allowed mass range. In the region where the rate is small enough for a freeze-out of a baryon asymmetry the one-loop correction is very small. However, this mass range is excluded by LEP data. Therefore, the baryon asymmetry of the universe cannot be understood in the minimal standard model with one Higgs doublet just as a $\mathrm{B}+\mathrm{L}$ asymmetry. The sphaleron rate in the experimentally allowed Higgs mass range is so much larger than the level required for $\mathrm{B}+\mathrm{L}$ freeze-out that higher loop, or non-perturbative effects should not be expected to rescue the baryon asymmetry. Extensions of the model have to be considered.

The major result of this paper is a systematic treatment of radiative corrections to the sphaleron rate close to the critical temperature where washing-out of a baryon asymmetry would be most severe. Previous studies of radiative corrections do not apply in this temperature range because their effective 3 -dimensional gauge coupling diverges at the lower critical temperature, and the whole scheme breaks down (no matter how small $\lambda$ is). This does not mean that sphaleron transitions actually become unsuppressed at $T_{0}$, but simply arises from choosing an unfortunate scheme for defining the coupling, not taking into account that we deal with a first order transition. Qualitatively, this is quite obvious. We have given a 
systematic framework for doing the quasiclassical expansion in this case. For $T_{0}<T<T_{c}$ there are no problems with a complex perturbative potential. In the 't Hooft - Feynman background gauge all fluctuations including Goldstone and Higgs fields have positive mass squared for all non-zero $\phi$ (compare eq. (3.13)), in this temperature range.

Similar considerations apply to the bounce solution describing the formation of critical bubbles at the phase transition. In this case the $\phi^{3}$ term is even more important because no bounce solution exists without it. Our approach will be applied to bubble formation in a forthcoming publication [36].

\section{ACKNOWLEDGMENTS}

We would like to thank Ch. Wetterich for useful discussions, and J. Baacke for a number of clarifying communications. 


\section{REFERENCES}

[1] G. 't Hooft, Phys. Rev. Lett. 37 (1976) 8; Phys. Rev. D14 (1976) 3432

[2] A. Ringwald, Nucl. Phys. B330 (1990) 1

[3] O. Espinosa, Nucl. Phys. B343 (1990) 310

[4] V. A. Kuzmin, V. A. Rubakov and M. E. Shaposhnikov, Phys. Lett. 155B (1985) 36

[5] N. Manton, Phys. Rev. D28 (1983) 2019;

F. R. Klinkhamer and N. S. Manton, Phys. Rev. D30 (1984) 2212

[6] A. D. Sakharov, JETP Lett. 6 91967) 24

[7] A. G. Cohen, D. B. Kaplan and A. E. Nelson, preprint UCSD-PTH-93-02; Ann. Rev. of Nucl. and Particle Sciences, Vol. 43, and references quoted therein

[8] P. Arnold and L. McLerran, Phys. Rev. D36 (1987) 581; D37 (1988) 1020

[9] M. Dine, R. Leigh, P. Huet, A. Linde and D. Linde, Phys. Rev. D46 (1992) 550

[10] G. W. Anderson and L. Y. Hall, Phys. Rev. D45 (1992) 2685

[11] M. E. Carrington, Phys. Rev. D45 (1992) 2933

[12] C. G. Boyd, D. E. Brahm and S. D. H. Hsu, preprint Calt-68-1795 (HUTP-92-A027, EFI-92-22)

[13] W. Buchmüller, Z. Fodor, T. Helbig and D. Walliser, preprint DESY 93-021

[14] J. R. Espinosa, M. Quiros and F. Zwirner, preprint CERN-TH-6577/92

[15] M. E. Shaposhnikov, Nucl. Phys. B287 (1987) 757; B299 (1988) 797;

A. I. Bochkarev, S. Yu. Khlebnikov ans M. E. Shaposhnikov, Nucl. Phys. B 329 (1990) 490

[16] L. Carson and L. McLerran, Phys. Rev. D41 (1990) 647

[17] L. Carson, X. Li, L. McLerran and R. Wang, Phys. Rev. D42 (1990) 2127

[18] B. Bunk, E. M. Ilgenfritz, J. Kripfganz and A. Schiller, Phys. Lett. B284 (1992) 371; Nucl. Phys. B403 (1993) 453.

[19] K. Kajantie, K. Rummukainen and M. Shaposhnikov, preprint CERN-TH 6901/93

[20] G. R. Farrar and M. E. Shaposhnikov, preprint CERN TH 6732/92; Phys. Rev. Lett. 70 (1993) 2833

[21] N. Turok, Phys. Rev. Lett. 68 (1992) 1803; preprint Imperial TP/91-92/33 and references quoted therein

[22] C. Wetterich, Nucl. Phys. B352 (1991) 529; Phys. Lett. B301 (1993) 90;

M. Reuter and C. Wetterich, preprint DESY 92-037/HD-THEP-92-62;

M. Reuter, N. Tetradis and C. Wetterich, preprint DESY 93-004/HD-THEP 93-2

[23] U. Ellwanger, preprrint HD-THEP 92-33, Z. f. Physik, to appear

[24] M. Hellmund, J. Kripfganz and M. G. Schmidt, Heidelberg preprint HD-THEP-93-23, June 1993

[25] J. Baacke and S. Junker, Phys.Rev. D49 (1994) 2055

[26] J. Baacke and S. Junker, Dortmund Preprint DO-TH-93/19EA, February 1994

[27] N. Tetradis and C. Wetterich, preprint DESY 92-093

[28] J. S. Langer, Physica 73 (1974) 61

[29] I. Affleck, Phys. Rev. Lett. 46 (1981) 388

[30] A. Ringwald, Phys. Lett. 201 (1988) 510

[31] J. Kripfganz and A. Ringwald, Z. Phys. C44 (1989) 213

[32] Y. Brihaye and J. Kunz, Phys. Lett. B249 (1990) 90

[33] L. Carson, Phys. Rev. D42 (1990) 2853 
[34] M .G. Schmidt and C. Schubert, preprint HD-THEP 93-24; Phys.Lett.B318 (1993) 438

[35] S. Braiband, Y. Brihaye and J. Kunz, Univ. Utrecht preprint THU-93/01

[36] J. Kripfganz, A. Laser and M. G. Schmidt, in preparation 


\section{FIGURES}

FIG. 1. The accessible range of $\lambda_{T} / g^{2}$ at $T_{0}$, for various values of the top quark mass.

FIG. 2. $A\left(\lambda_{T} / g^{2}, \epsilon(T)\right)$ as function of $\lambda_{T} / g^{2}$.

FIG. 3. Various contributions to the sphaleron action.

FIG. 4. $S_{F F}^{(1, \beta)}$ relative to the leading contribution. 


\section{TABLES}

TABLE I. The first few operators required for the proper time expansion (3.7) are evaluated for the sphaleron background field, for two values of $\frac{\lambda_{T}}{g^{2}}$. The operators $V_{i}$ are the ones listed in Appendix A of ref. [16].

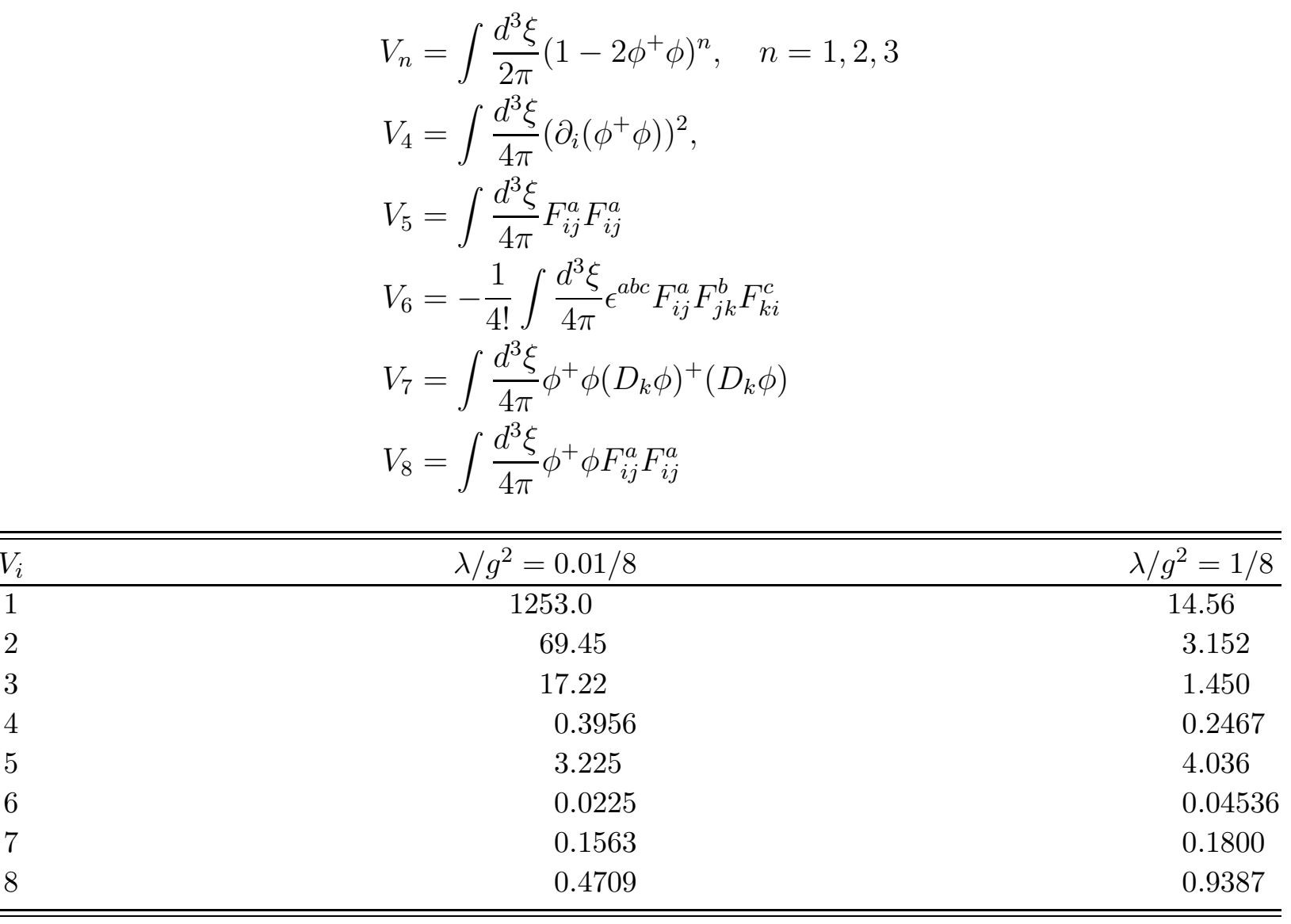

